\begin{tabular}{|lll} 
SARIPEX & ORIGINAL RESEARCH PAPER & Social Science \\
\cline { 2 - 3 } & $\begin{array}{l}\text { PERCEPTION OF PARENTS ABOUT THE } \\
\text { VIRTUAL LEARNING OF THEIR CHILDREN }\end{array}$ & $\begin{array}{l}\text { KEY WORDS: Virtual } \\
\text { DURING COVIning, Working Parents, } \\
\text { STUDY DESIGN. }\end{array}$ \\
\hline
\end{tabular}

\section{Dr. A.Arul Paul}

Dr. Srinivas.G
Ph.D (Social Work), Social Welfare Officer, The Tamil Nadu Dr. M.G.R Medical University, 69, Anna Salai, Guindy Chennai -32.

M.D, Prof and HOD, Department of Epidemiology, The Tamil Nadu Dr. M.G.R Medical University, 69, Anna Salai, Guindy Chennai -32.

\section{Dr. Parameswari} .S

Associate Professor, Department of Epidemiology, The Tamil Nadu Dr. M.G.R Medical University, 69, Anna Salai, Guindy Chennai-32.

\section{Dr. Jasmine S.} Sundar
Assistant Professor, Department of Epidemiology, The Tamil Nadu Dr. M.G.R Medical University, 69, Anna Salai, Guindy Chennai -32.

Background: The Covid-19 pandemic has brought a drastic change in the educational system in India. The students are out of classroom setup in the world, which resulting technology has emerged as a successful milestone where school has adopted digital platforms for teaching. This Research studies aimed at the perception of parents about the virtual E- learning of their children during covid-19 pandemic situation and the study was a mixed study design $(\mathrm{N}=87)$ with a self-

0 administered questionnaire. Descriptive statistics was done by using SPSS ver-25. Majority of the population was female and the mean age was 41-50 years. Children were attended maximum 4-hours online education with the support of mobile phone but the parents were reported that their inability to monitor their children academic role during the online services. Though the school has a separate app to cover the requirements of children's need but the quality of the content of the curriculum is questioned. Children were suffering with irritation of the eyes, behavioral problems, and parents are becoming an expert in providing counseling. Parents were reported of e-learning should help the weaker students and support them by providing regular feedback. This study concludes that parents were agreed the online education during this pandemic, but they were a highly preferred traditional school of practice.

\section{INTRODUCTION:}

Covid -19 has caused in closure of schools across the world. The school education has changed forcefully, with the typical rise of e-learning, whereby education is undertaken remotely and on digital platforms. Many researchers suggest that online learning platform has been revealed to increase retention of information, and take less time, import the changes corona virus have caused might be here to stay. (1) The need for learning has been increased with the rapid growth of modern technology and knowledge, but the speed and volume of learning are fluctuating. The main challenge that we need to overcome is how to acquire knowledge and skills along with the organization of learning resources to keep up knowledge economy. (2)

As a result, technology integration in education has emerged as a successful milestone of imparting quality teaching and educational content. The e-Learning is not about incorporating if technology in schools so that it can replace teachers with the technological gadgets. Moreover, for the betterment of the student's e-learning is now incorporated as an essential part of student learning. The children of parents have faced a lot of obstacles and challenges during the motivation for their children to participate in the one-line education. The majority of the Indian parents have felt that the e-teaching is not as much as better than traditional teaching and the quality of e-teaching is not satisfactory as compared from Government schools to Private Schools, and the impact of e-learning is comparatively less. (3)

\section{Review Of Related Literature:}

Showkat Ahmad Dar et al., (2020) did a study on Impact of Covid 19 on Education in India. This covid -19 circumstance challenged the education system across the world and forced educators to shift to an online mode of teaching overnight. Most of the academic institutions that were previous averse to change their traditional academic method had no choice but to shift completely to online education. (4)

Eva Yi Hung Lau et al., conducted a study (2021) on online www.worldwidejournals.com learning and parent's satisfaction during covid-19. The study information originated from an online survey conducted in Hong Kong in February 2020. The respondents were parents. The findings illustrated that equally the length of online learning and the amount of homework were related to the parents' satisfaction, but these relations were qualified by the children's ability as more competent in engaging in online learning independently. The results recommend that in manipulative online learning, thought of children's capacity to complete such learning self-reliantly will help increase parents' satisfaction. (5)

Sonia Zaccoletti et al., (2020) did a study of Parents' Perceptions of Student Academic Motivation during the COVID-19 Lockdown: A Cross-Country Comparison (Italian and Portuguese). Consequently a retrospective pretest and posttest design, $($ Italy $=173$, Portugal $=394) 567$ parents have stated on their child's academic inspiration and involvement in extracurricular activities (grades 1 to 9). The researcher was used a multi-group latent change score model to compare motivation scores on pre covid mean; rate of change; individual variation change \& dependence of the rate of change on initial motivation. The study outcomes were also quantified that the deterioration in the student's involvement in extracurricular activities were related with changes in academic motivation. Moreover, schoolchildren age was significantly associated with changes in motivation. No significant associations were found for student's gender or in a parent's education. The research study thus, resists that teachers need to adopt motivation-enhancing practices as a means to prevent the deterioration in academic motivation during exceptional situations (6).

Ifoni Ludji et al., did a study (2021) on parent's perception on the implementation of home learning during covid-19. Descriptive qualitative study design was taking as a case in two elementary schools in Kupang, East Nusa Tenggara. There were 20 parents from both schools taken purposefully as the sample. 20 (twenty) questions posted on Google Form as the tool. The results showed that the execution of home learning 
brought two perceptions of the benefits and challenges. The benefits were concerned the possibility of home learning to provide a personalized experience and flexible system of education, to minimize the possibility of the children to get infected by the Covid-19. Further perception stated that the execution of home learning also brought certain challenges to those parents such as, demanding more expenses to buy the internet quota, causing the children less motivated and irresistible in learning, hovering the work load of the parents, time inadequacy for working parents by spending more time to assist the kids during the learning, increasing the tense between the kids and parents during the learning (7).

Andre Hasudungan Lubis et al., (2020) were done a study of Parents Perceptions on e-learning during covid-19 pandemic in Indonesia. Totally 257 participants were asked about this issue by using a web-based survey and the descriptive data are used for the analysis of the study. Outcomes specified that participants were overall dissatisfied with the execution of elearning during the pandemic. Parents demanded that the poor of ICT infrastructure (e.g. the digital devices, Internet, electricity) and lack of technology skills become the barriers for their children to use e-learning. Parents also considered that traditional learning is desirable to online learning (8).

\section{Rationale ForThe Study:}

The Digital divides between parents who can manage and who cannot be manage their child's online education. Many Indian families in the urban areas are living in a nuclear family setup and almost all women in the family are engaged in employment, and unable to supervise their child's online classes, finds difficult to dedicate a few hours daily for the educational growth of their kids. (9)

The child with different learning abilities is a great challenge for the parents and hence it's required their involvement to manage the diversity of learners' children who want to learn at their own pace.

Though the teaching and learning process becomes an enjoyable activity for parents, but at the same time it also raises their blood pressure every day because of families are struggling every day with growing demand for personal space and individual gadgets and parents' involvement must be much more than the students' participation. The parents are needed to emphasize skills in logical thinking, critical analysis and problem-solving assignments that require in helping their child's assignment. Their major task is to direct and self-motivate their ward to follow the guided instruction given by the teacher. (10)

Suddenly jumping into online education can be intimidating and confusing for first time online students and any break in the data connectivity can cause a lack of continuity in the learning for the child, which can be harmful, and parents does not aware of how to solve such issues. This abrupt transition to online hardly compensates for the absence of classroom experiences, lack of social interaction, health hazards of staring at a screen continuously for long periods.

Many working parents feel that face to face relationships are difficult to foster in the digital world e-learning methods of education which are critically needed in the teachinglearning activities. This e-learning mode of education delivers only academic content. The physical, social and emotional needs of the children/learner go undetected when there is no physical and visual contact between the teacher and the student. (11)

Children's use of the Internet has some positive effects such as access to information, communication, and support for education and individual development. Additionally, it is asserted that children's Internet use also has some negative effects such as exposure to violence and pornography. (12)
UNESCO (2021) Parent participation is connected to children's total learning during the school shutdown is important to monitor their children not to get involved into a risky behaviours due to peer group pressures and substance abuse.(13)

\section{Primary Objective:}

To asses parents understanding and Perception about the virtual learning of their children during COVID-19 pandemic.

\section{Secondary Objectives:}

1. To explore the parents' involvement of their child'se-learning.

2. To assess the parents' perception of e-learning implemented by schools.

3. To study the issues faced by the parents' due to e-learning of their children.

4. To find out the ways to promote e-learning.

\section{METHODS AND DISCUSSION:}

Study Place:

The Tamil Nadu Dr. MGR Medical University

\section{Study Population:}

Parents of all students from classes $1^{\text {st }}$ to $12^{\text {th }}$ standard

\section{Inclusion Criteria:}

- Parents of all students from classes $1^{\text {st }}$ to $12^{\text {th }}$ standard

- Parents' in all categories.

Study Period:

February 2021 to May 2021 (4 months Duration)

\section{Sample Size:}

All parents who was eligible and willing to participate was enrolled into the study.

\section{Study Design:}

The researcher had adopted Mixed Study Design.

Data Collection Procedure: The Researcher had adopted a Total Sampling Method for his study, because the target group is small and well-defined characteristic. The initial tool developed was finalized after conduct a pilot study and no changes were taken in the questionnaire. The collected data were entered into Microsoft-excel and analyzed by using SPSS Software.

\section{RESULTS:}

Table No: 1 Socio-Demographic Details Of The Working Parents.

\begin{tabular}{|c|c|c|}
\hline Variables & Frequency & Percentage \\
\hline \multicolumn{3}{|l|}{ Age Distribution (Years) } \\
\hline $30-35$ & 05 & 05.7 \\
\hline $36-40$ & 19 & 21.8 \\
\hline $41-45$ & 27 & 31.0 \\
\hline $46-50$ & 30 & 34.5 \\
\hline $51-55$ & 05 & 05.7 \\
\hline $56-60$ & 01 & 01.1 \\
\hline \multicolumn{3}{|l|}{ Gender } \\
\hline Male & 23 & 26.4 \\
\hline Female & 64 & 73.6 \\
\hline \multicolumn{3}{|l|}{ Educational Qualification } \\
\hline Primary & 00 & 00.0 \\
\hline Secondary & 06 & 06.9 \\
\hline Higher Secondary & 00 & 00.0 \\
\hline Degree & 74 & 85.1 \\
\hline Any Other & 7 & 08.0 \\
\hline Illiterate & 00 & 00.0 \\
\hline \multicolumn{3}{|l|}{\begin{tabular}{|l|} 
Designation \\
\end{tabular}} \\
\hline Sergeant & 01 & 1.1 \\
\hline Assistant & 29 & 33.3 \\
\hline Assistant Section Officer & 24 & 27.6 \\
\hline Section Officer & 02 & 2.3 \\
\hline
\end{tabular}


PARIPEX - INDIAN JOURNAL OF RESEARCH | Volume - 11 | Issue - 02 |February - 2022 | PRINT ISSN No. 2250 - 1991 | DOI : $10.36106 /$ paripex

\begin{tabular}{|l|l|l|}
\hline Lab Technician & 07 & 8.0 \\
\hline Nurses & 01 & 1.1 \\
\hline Personal Assistant & 07 & 8.0 \\
\hline Office Assistant & 03 & 3.4 \\
\hline Social Welfare Officer & 02 & 2.3 \\
\hline Record Clerk & 03 & 3.4 \\
\hline Computer Operator & 04 & 4.6 \\
\hline Programmer & 02 & 2.3 \\
\hline Junior Engineer & 01 & 1.1 \\
\hline Biochemists' & 01 & 1.1 \\
\hline Gender of School Children & \\
\hline Male & 50 & 57.5 \\
\hline Female & 37 & 42.5 \\
\hline Educational Status of present school going children. \\
\hline Primary & 16 & 18.3 \\
\hline Elementary & 22 & 25.2 \\
\hline Secondary & 17 & 19.5 \\
\hline Higher Secondary & 32 & 36.7 \\
\hline
\end{tabular}

\begin{tabular}{|l|l|l|}
\hline Husband & 9 & 10.3 \\
\hline Grand Parents & 21 & 24.1 \\
\hline Elder siblings & 09 & 10.3 \\
\hline Self (no one supervise) & 36 & 41.4 \\
\hline Total & 87 & 100.0 \\
\hline
\end{tabular}

Table No.5 Parent's Involvement In E- Learning (Through Observation)

\begin{tabular}{|c|c|c|c|c|c|}
\hline \multirow{2}{*}{\begin{tabular}{|l|} 
Parents Perception on \\
Educational Growth of \\
Children through Virtual \\
Mode
\end{tabular}} & \multicolumn{5}{|c|}{ Frequencies } \\
\hline & \begin{tabular}{|l|} 
Strong \\
ly \\
Disagr \\
ee \\
f $(\%)$
\end{tabular} & \begin{tabular}{l|} 
Disa \\
gree \\
f $(\%)$
\end{tabular} & $\begin{array}{l}\text { Unde } \\
\text { cide } \\
\text { d } \\
\text { f }(\%)\end{array}$ & \begin{tabular}{|l|} 
Agr \\
ee \\
f (\%)
\end{tabular} & $\begin{array}{l}\text { Stron } \\
\text { gly } \\
\text { Agre } \\
\text { e } \\
\text { f }(\%)\end{array}$ \\
\hline $\begin{array}{l}\text { Parents concur on to build } \\
\text { a rapport with child's } \\
\text { teacher for the growth and } \\
\text { development for them } \\
\text { during the offline Virtual } \\
\text { courses. }\end{array}$ & $\begin{array}{l}04 \\
(04.6 \%)\end{array}$ & \begin{tabular}{|l|}
10 \\
$(11.5$ \\
$\%)$
\end{tabular} & $\begin{array}{l}20 \\
(23.0 \\
\%)\end{array}$ & \begin{tabular}{|l|}
35 \\
$(40$. \\
$2 \%)$ \\
\end{tabular} & $\begin{array}{l}18 \\
(20.7 \\
\%)\end{array}$ \\
\hline $\begin{array}{l}\text { e-learning has proved a } \\
\text { boom while allowing } \\
\text { students to interact with } \\
\text { the teacher as they feel } \\
\text { like a school environment }\end{array}$ & \begin{tabular}{|l|}
12 \\
$(13.8 \%)$
\end{tabular} & \begin{tabular}{|l|}
35 \\
$(40.2$ \\
$\%)$
\end{tabular} & $\begin{array}{l}15 \\
(17.2 \\
\%)\end{array}$ & \begin{tabular}{|l|}
22 \\
$(25$. \\
$3 \%)$ \\
\end{tabular} & $\begin{array}{l}03 \\
(03.4 \\
\%)\end{array}$ \\
\hline $\begin{array}{l}\text { Teacher's appropriate, } \\
\text { helpful feedback will } \\
\text { enhance the children } \\
\text { educational growth. }\end{array}$ & \begin{tabular}{|l}
06 \\
$(06.9$
\end{tabular} & \begin{tabular}{|l|}
14 \\
$(16.1$ \\
$\%)$
\end{tabular} & $\begin{array}{l}12 \\
(13.8 \\
\%)\end{array}$ & \begin{tabular}{|l|}
50 \\
$(57$. \\
$5 \%)$ \\
\end{tabular} & $\begin{array}{l}05 \\
(05.7 \\
\%)\end{array}$ \\
\hline $\begin{array}{l}\text { An online curricular } \\
\text { program developed by } \\
\text { school is visually pleasin } \\
\end{array}$ & $\begin{array}{l}09 \\
(10.3 \%)\end{array}$ & \begin{tabular}{|l|}
26 \\
$(29.9$ \\
$\%)$ \\
\end{tabular} & \begin{tabular}{|l|}
19 \\
$(21.8$ \\
$\%)$ \\
\end{tabular} & \begin{tabular}{|l|}
29 \\
$(33$. \\
$3 \%)$ \\
\end{tabular} & \begin{tabular}{|l|}
04 \\
$(04.6$ \\
$\%)$ \\
\end{tabular} \\
\hline $\begin{array}{l}\text { Parents opinion about the } \\
\text { Educational content of the } \\
\text { online curriculum } \\
\text { programs developed by } \\
\text { school is of high quality. }\end{array}$ & \begin{tabular}{|l|}
12 \\
$(13.8 \%)$
\end{tabular} & \begin{tabular}{|l|}
38 \\
$(43.7$ \\
$\%)$
\end{tabular} & \begin{tabular}{|l}
25 \\
$(28.7$ \\
$\%)$
\end{tabular} & \begin{tabular}{|l|}
10 \\
$(11$. \\
$5 \%)$ \\
\end{tabular} & $\begin{array}{l}02 \\
(02.3 \\
\%)\end{array}$ \\
\hline
\end{tabular}

Table No.2 Number Of Living Rooms Available In The Respondents' Residence

\begin{tabular}{|l|l|l|}
\hline Number of Living Rooms & $\begin{array}{l}\text { No. of } \\
\text { Respondents }\end{array}$ & Percentage \\
\hline One room & 29 & 33.3 \\
\hline Two rooms & 47 & 54.0 \\
\hline Three rooms & 11 & 12.7 \\
\hline Total & 87 & 100.0 \\
\hline
\end{tabular}

Table No.3 Internet Connection In The Home Before COVID Pandemic.

\begin{tabular}{|l|l|l|}
\hline Internet Facilities & No. of Respondents & Percentage \\
\hline Yes & 16 & 18.4 \\
\hline No & 71 & 81.6 \\
\hline Total & 87 & 100.0 \\
\hline
\end{tabular}

Tableno.4 Parent's Involvement In E- Learning (Through Physical)

\begin{tabular}{|c|c|c|c|c|c|}
\hline \multirow[b]{2}{*}{\begin{tabular}{|l|} 
Parents \\
Physical \\
Involveme \\
nt in \\
Children \\
Education
\end{tabular}} & \multicolumn{5}{|c|}{ Frequencies } \\
\hline & \begin{tabular}{|l} 
Always \\
f (\%)
\end{tabular} & $\begin{array}{l}\text { Often } \\
\text { f (\%) }\end{array}$ & $\begin{array}{l}\text { Sometimes } \\
\text { f (\%) }\end{array}$ & $\begin{array}{l}\text { Rarely } \\
\text { f (\%) }\end{array}$ & $\begin{array}{l}\text { Never } \\
\text { f (\%) }\end{array}$ \\
\hline $\begin{array}{l}\text { Check the } \\
\text { requirement } \\
\text { s before } \\
\text { online } \\
\text { classes. }\end{array}$ & $\begin{array}{l}19 \\
(21.8 \%)\end{array}$ & \begin{tabular}{|l|}
23 \\
$(26.4 \%)$
\end{tabular} & \begin{tabular}{|l|}
32 \\
$(36.8 \%)$
\end{tabular} & $\begin{array}{l}06 \\
(06.9 \%)\end{array}$ & $\begin{array}{l}07 \\
(08.0 \%)\end{array}$ \\
\hline $\begin{array}{l}\text { Ensure the } \\
\text { designated } \\
\text { place }\end{array}$ & \begin{tabular}{|l|}
32 \\
$(36.8 \%)$
\end{tabular} & \begin{tabular}{|l}
18 \\
$(20.7 \%)$
\end{tabular} & \begin{tabular}{|l|}
29 \\
$(33.3 \%)$
\end{tabular} & \begin{tabular}{|l|}
02 \\
$(02.3 \%)$
\end{tabular} & \begin{tabular}{|l}
06 \\
$(06.9 \%)$
\end{tabular} \\
\hline $\begin{array}{l}\text { Parents } \\
\text { familiarize } \\
\text { the e- } \\
\text { learning }\end{array}$ & $\begin{array}{l}17 \\
(19.5 \%)\end{array}$ & $\begin{array}{l}06 \\
(06.9 \%)\end{array}$ & \begin{tabular}{|l|}
23 \\
$(26.4 \%)$
\end{tabular} & $\begin{array}{l}10 \\
(11.5 \%)\end{array}$ & $\begin{array}{l}31 \\
(35.6 \%)\end{array}$ \\
\hline \begin{tabular}{|l|}
$\begin{array}{l}\text { Monitor the } \\
\text { internet use }\end{array}$ \\
\end{tabular} & \begin{tabular}{|l|}
27 \\
$(31.0 \%)$ \\
\end{tabular} & \begin{tabular}{|l}
13 \\
$(14.9 \%)$
\end{tabular} & \begin{tabular}{|l|}
39 \\
$(44.8 \%)$ \\
\end{tabular} & \begin{tabular}{|l|}
06 \\
$(06.9 \%)$
\end{tabular} & \begin{tabular}{|l|}
02 \\
$(02.3 \%)$
\end{tabular} \\
\hline \begin{tabular}{|l|} 
Monitor \\
their child's \\
Performance \\
\end{tabular} & $\begin{array}{l}23 \\
(26.4 \%)\end{array}$ & \begin{tabular}{|l|}
21 \\
$(24.1 \%)$
\end{tabular} & $\begin{array}{l}35 \\
(40.2 \%)\end{array}$ & $\begin{array}{l}06 \\
(06.9 \%)\end{array}$ & \begin{tabular}{|l}
02 \\
$(02.3 \%)$
\end{tabular} \\
\hline $\begin{array}{l}\text { Involvement } \\
\text { in helping } \\
\text { to complete } \\
\text { the child's } \\
\text { home work. }\end{array}$ & $\begin{array}{l}19 \\
(21.8 \%)\end{array}$ & $\begin{array}{l}17 \\
(19.5 \%)\end{array}$ & \begin{tabular}{|l}
35 \\
$(40.2 \%)$
\end{tabular} & $\begin{array}{l}06 \\
(06.9 \%)\end{array}$ & $\begin{array}{l}10 \\
(11.5 \%)\end{array}$ \\
\hline
\end{tabular}

Table No.6 Supervise The Children Attended The Online Classes By The Family Members.

\begin{tabular}{|l|l|l|}
\hline $\begin{array}{l}\text { Supervise the child } \\
\text { attended the online classes }\end{array}$ & $\begin{array}{l}\text { No. of } \\
\text { Respondents }\end{array}$ & Percentage \\
\hline Wife & 12 & 13.8 \\
\hline
\end{tabular}

Table No.7 Parents' Opinion On Execute The Virtual Learning Platform By School During Covid-19 Pandemic.

Questions \begin{tabular}{|l|l|} 
Frequencies & Percentage \\
\hline
\end{tabular}

Types of School

\begin{tabular}{|l|l|l|}
\hline Public & 07 & 08.0 \\
\hline Private & 80 & 92.0
\end{tabular}

School have a separate app to connect the online programme

\begin{tabular}{|l|l|l|}
\hline Yes & 65 & 74.7 \\
\hline No & 22 & 25.2 \\
\hline
\end{tabular}

School regularly conducted the online classes

\begin{tabular}{|l|l|l|}
\hline Yes & 83 & 95.4 \\
\hline No & 04 & 04.6 \\
\hline
\end{tabular}

Duration of the children engaged in the online class.

\begin{tabular}{|l|l|l}
\hline Continuously an hour & 02 & 02.3
\end{tabular}

\begin{tabular}{|l|l|l|}
\hline 2 hours & 11 & 12.6 \\
\hline 3 hours & 20 & 23.0 \\
\hline 4 hours & 41 & 47.1 \\
\hline Full Day & 13 & 15.0 \\
\hline Not at all & 00 & 00.0
\end{tabular}

Teacher able to cover all the chapters

\begin{tabular}{|l|l|l|}
\hline Yes & 37 & 42.5 \\
\hline No & 50 & 57.4 \\
\hline
\end{tabular}

Methods used by school monitors the children performance.

\begin{tabular}{|l|l|l|}
\hline Regular Google home work & 28 & 32.1 \\
\hline
\end{tabular}

\begin{tabular}{|l|l|l|}
\hline Leisure time activities & 03 & 03.4 \\
\hline
\end{tabular}

\begin{tabular}{|l|l|l}
\hline Conduct the period assessment & 17 & 19.5
\end{tabular}

\begin{tabular}{|l|l|l}
\hline Submit online assignment & 17 & 19.5
\end{tabular}

\begin{tabular}{|l|l|l|}
\hline Conduct online exhibition & 04 & 04.5
\end{tabular}

\begin{tabular}{|l|l|l|}
\hline All the above & 18 & 20.6 \\
\hline
\end{tabular}

www.worldwidejournals.com 


\begin{tabular}{|c|c|c|}
\hline None of the above & 00 & 000 \\
\hline \multicolumn{3}{|c|}{ Children talented to follow the Teachers instruction } \\
\hline Always & 28 & 32.1 \\
\hline Often & 39 & 44.8 \\
\hline Sometimes & 15 & 17.2 \\
\hline Rarely & 04 & 04.6 \\
\hline Never & 01 & 01.1 \\
\hline \multicolumn{3}{|c|}{$\begin{array}{l}\text { Children smart to get clarification of their doubt during e- } \\
\text { learning }\end{array}$} \\
\hline Always & 11 & 12.6 \\
\hline Often & 27 & 31.0 \\
\hline Sometimes & 31 & 35.6 \\
\hline Rarely & 10 & 11.4 \\
\hline Never & 08 & 09.2 \\
\hline \multicolumn{3}{|c|}{$\begin{array}{l}\text { Teachers used blackboard or any materials during online } \\
\text { classes. }\end{array}$} \\
\hline Always & 27 & 31.0 \\
\hline Often & 31 & 35.6 \\
\hline Sometimes & 17 & 19.5 \\
\hline Rarely & 08 & 9.2 \\
\hline Never & 04 & 4.6 \\
\hline
\end{tabular}

Table.8 Difficulties Faced By Parents Due To Online Classes During Covid-19

\section{Questions}

Frequencies Percentage

Obstacles faced by the Parents during the online classes

\begin{tabular}{|l|l|l|}
\hline Exacerbate / Bad situation & 12 & 13.6 \\
\hline Technical Issues & 41 & 47.0 \\
\hline Computer Literacy & 04 & 4.5 \\
\hline Time Management & 25 & 28.7 \\
\hline Self-Motivation & 05 & 5.7 \\
\hline \multicolumn{2}{|l|}{ Health Issues faced by the school children } \\
\hline Sleep - Disturbances & 20 & 22.9 \\
\hline Eating Disorders & 10 & 11.4 \\
\hline Behavioral Problems & 22 & 25.2 \\
\hline Irritation of eyes & 28 & 32.1 \\
\hline Hearing Problems & 07 & 08.0 \\
\hline
\end{tabular}

Parents thoughts in handle the distress situation among their children

\begin{tabular}{|l|l|l|}
\hline Physical activities & 35 & 40.2 \\
\hline Peeping the child & 05 & 05.7 \\
\hline Using harsh words & 03 & 03.4 \\
\hline Positive reinforcement & 24 & 27.5 \\
\hline Plan a work schedule & 04 & 04.5 \\
\hline Being a facilitator & 03 & 03.4 \\
\hline $\begin{array}{l}\text { Help to complete daily home } \\
\text { work }\end{array}$ & 13 & 14.9 \\
\hline
\end{tabular}

Difficulties in balancing the office work, household responsibilities, and teaching to their children

\begin{tabular}{|l|l|l|}
\hline Always & 35 & 40.2 \\
\hline Often & 15 & 17.2 \\
\hline Sometimes & 28 & 32.2 \\
\hline Rarely & 05 & 05.7 \\
\hline Never & 04 & 04.6
\end{tabular}

Parents immediate reactions towards their children misses the online education

\begin{tabular}{|l|l|l|}
\hline Scolding & 20 & 22.9 \\
\hline Scolding and beating & 09 & 10.3 \\
\hline Severe physical punishment & 02 & 2.3 \\
\hline Counseling & 45 & 51.7 \\
\hline Others & 11 & 12.6
\end{tabular}

Steps taken by parents to improve their children studies

Allow for group study

Arrange tuition 09

Extra care will be provided

23

10.2

Leave the children as they like 08

26.4

\begin{tabular}{l|l|l|}
\hline like & 08 & 9.2
\end{tabular} site

Advice

\begin{tabular}{|l|l|l|}
\hline Lock the site & 20 & 23.0 \\
\hline Scolding and beating & 13 & 14.9 \\
\hline Scolding and also lock the site & 2 & 2.3 \\
\hline Strict Monitor & 2 & 2.3 \\
\hline No way (leave them) & 1 & 1.1 \\
\hline
\end{tabular}

Table No.9 Parental Thoughts On Ways To Promote Of ELearning

\begin{tabular}{|c|c|c|c|c|c|}
\hline \begin{tabular}{|l|} 
Parental \\
feelings on \\
ways to \\
promote of e- \\
learning in \\
schools
\end{tabular} & \begin{tabular}{|l|} 
Strongly \\
Disagre \\
e
\end{tabular} & \begin{tabular}{|l|} 
Disagr \\
ee
\end{tabular} & $\begin{array}{l}\text { Undeci } \\
\text { ded }\end{array}$ & Agree & $\begin{array}{l}\text { Strongl } \\
\text { y Agree }\end{array}$ \\
\hline $\begin{array}{l}\text { Build a } \\
\text { personal } \\
\text { connection } \\
\text { with students }\end{array}$ & \begin{tabular}{|l}
21 \\
$(24.13)$
\end{tabular} & \begin{tabular}{|l|}
12 \\
$(13.79)$
\end{tabular} & \begin{tabular}{|l|}
06 \\
$(6.89)$
\end{tabular} & \begin{tabular}{|l}
44 \\
$(50.57)$
\end{tabular} & $\begin{array}{l}04 \\
(4.59)\end{array}$ \\
\hline $\begin{array}{l}\text { Motivate the } \\
\text { students }\end{array}$ & \begin{tabular}{|l}
23 \\
$(26.43)$
\end{tabular} & $\begin{array}{l}07 \\
(8.04)\end{array}$ & $\begin{array}{l}03 \\
(3.44)\end{array}$ & $\begin{array}{l}36 \\
(41.37)\end{array}$ & \begin{tabular}{|l|}
8 \\
$(20.68)$
\end{tabular} \\
\hline $\begin{array}{l}\text { Help students } \\
\text { to maintain } \\
\text { focus }\end{array}$ & $\begin{array}{l}21 \\
(24.13)\end{array}$ & $\begin{array}{l}06 \\
(6.8)\end{array}$ & $\begin{array}{l}08 \\
(9.17)\end{array}$ & $\begin{array}{l}38 \\
(43.67)\end{array}$ & $\begin{array}{l}14 \\
(16.09)\end{array}$ \\
\hline $\begin{array}{l}\text { Create a sense } \\
\text { of community } \\
\text { feelings }\end{array}$ & \begin{tabular}{|l}
22 \\
$(25.28)$
\end{tabular} & $\begin{array}{l}07 \\
(8.04)\end{array}$ & $\begin{array}{l}16 \\
(18.39)\end{array}$ & $\begin{array}{l}31 \\
(35.63)\end{array}$ & $\begin{array}{l}11 \\
(12.64)\end{array}$ \\
\hline $\begin{array}{l}\text { Make a } \\
\text { meaning } \\
\text { discussion }\end{array}$ & $\begin{array}{l}23 \\
(26.43)\end{array}$ & $\begin{array}{l}07 \\
(8.04)\end{array}$ & $\begin{array}{l}05 \\
(5.74)\end{array}$ & $\begin{array}{l}39 \\
(44.82)\end{array}$ & $\begin{array}{l}13 \\
(14.94)\end{array}$ \\
\hline $\begin{array}{l}\text { Increase } \\
\text { student's } \\
\text { engagement }\end{array}$ & $\begin{array}{l}23 \\
(26.43)\end{array}$ & $\begin{array}{l}07 \\
(8.04)\end{array}$ & $\begin{array}{l}03 \\
(3.44)\end{array}$ & $\begin{array}{l}38 \\
(43.67)\end{array}$ & $\begin{array}{l}16 \\
(18.39)\end{array}$ \\
\hline $\begin{array}{l}\text { Identify and } \\
\text { support } \\
\text { struggling } \\
\text { students }\end{array}$ & \begin{tabular}{|l}
23 \\
$(26.43)$
\end{tabular} & \begin{tabular}{|l|}
08 \\
$(9.17)$
\end{tabular} & $\begin{array}{l}02 \\
(2.29)\end{array}$ & $\begin{array}{l}39 \\
(44.82)\end{array}$ & \begin{tabular}{|l|}
15 \\
$(17.24)$
\end{tabular} \\
\hline $\begin{array}{l}\text { Provide } \\
\text { regular feed } \\
\text { back }\end{array}$ & \begin{tabular}{|l}
22 \\
$(25.28)$
\end{tabular} & $\begin{array}{l}04 \\
(4.59)\end{array}$ & $\begin{array}{l}04 \\
(4.59)\end{array}$ & $\begin{array}{l}48 \\
(55.17)\end{array}$ & $\begin{array}{l}09 \\
(10.34)\end{array}$ \\
\hline
\end{tabular}

\section{DISCUSSION:}

- The mean age of the study population lay between 41-50 years.

- Majority (73.6\%) of the working parents were female.

- Half (56\%) of the working parents' children were studying secondary and higher secondary level of schooling.

- One third (33.3\%) of the parents were managed with a single living bed room which covers their child's education online requirements too.

- A vast majority $(81.6 \%)$ of the working parents did not have an internet facility before the covid-19 pandemic. But after covid-19 it was reversing down that the parents were forced to buy new android mobile phone with unlimited internet package for their children educational virtual learning purpose.

- Most (47\%) of the working parents did not familiarize themselves about the virtual learning platform, commented that regular school teaching was helping the children to acquire knowledge.

- Out of their tight scheduled, a high majority $(90.7 \%)$ of them were always, often and sometimes monitored their children internet usage.

- More than two third (71\%) of the children's parents were spending their precious time by assigning themselves in helping their children to complete their homework on time.

- The results signified that more than one third (35\%) of them were agreed that building a rapport with school teacher during the offline virtual courses for their growth and development.

- The results also indicated that one third $(35 \%)$ of the parents indicated that online education was not boomed as they felt as like a school environment. 
- It was noted that half (55\%) of the parent's perception was agreed that feedback given by the teachers during the online education would enhance their children educational growth.

- The results highlighted that half (50\%) of the parents did not agree that the educational content designed specially for the online curriculum by the school were not the highly excellent.

- The results signified that most (41.4\%) of the working parents have said that no one was supervised their children in the absence of them during the online education.

- The absolute majority (92\%) of the working parents' children were studying in private schools, but around one fourth $(25 \%)$ of the school did not have apps meet the child's educational requirement during the covid-19 pandemic.

- Less than half (47\%) of the school children were engaged virtual classes for more than 4 hours in a day. But half $(57 \%)$ of the parents were highlighted that the teachers did not cover all the subjects during the virtual classes.

- It has been cleared, that less than one fourth (32\%) of the schools had adopted the following methods (Regular Google homework, Leisure time activities, Conduct the period assessment, submit an online assignment and conduct online exhibition) to monitor the student's performance and based on that the progress report of the children were prepared.

- The majority $(67 \%)$ of the parents have said that the subject's teachers were using the black board / any kind of educational materials during the online classes for the better understanding of their children.

- The results represent that a considerable proposition $(13.6 \%)$ of the working parents was facing obstacles on exacerbate the situation during the online classes due to various factors prevailed in the home environment. The results also support that most $(47 \%)$ of the parents were facing technical issues and they needed technical person supports.

- Less than one third (32\%) of the parent's children were suffering with irritation of the eyes as a health issue, one fourth $(25 \%)$ of them were noticed that considerable changes in their behavior.

- The results emphasized that most $(40 \%)$ of the parents were motivated their children into various physical activities and introduced the positive reinforcement technique to reduce their stress. One forth $(27 \%)$ of the parents were handling their children through introduced positive reinforcement techniques to handle the distress.

- Half $(57.4 \%)$ of the working parents were truly found difficulty in balancing the work, household chores, and also taking care of their children educational activities.

- The results illuminated that one third $(32.2 \%)$ of the working parent's were scolding and beating towards their children missed out the online education, but at the same time half $(51.7 \%)$ of them were provide counseling to make them to understand the importance of the education and half (54\%) of the parents were providing an extra care to meet out their children academic activities, remaining of them were arranged tuition for them. Half (56.3\%) of them were provided advice to their children not to see the unwanted site.

- It has been revealed that half (55\%) of the working parents were agreed, to building a personal connection with students, but more than one third $(37.8 \%)$ of the working parents were disagreeing the statement of building a personal connection with students in order to promote elearning. One third (34\%) of them were not agreed the statement of motivating the students. Half $(59.6 \%)$ of the parent's perceptions were agreed that schools should provide special focus and attention to each individual's especially for weaker students. Most $(48.2 \%)$ of the parent's perceptions were that the school must create a sense of community feelings among the students to participate in the online education. Half $(59.7 \%)$ of the parents were agreed and said that school would create a pave to have a meaning discussion among the students during the online. The majority $(61.9 \%)$ of the parents were agreed that school environment could increase the student's engagement with their subject teachers during the online classes for their betterment of their educational standard. The majority $(62 \%)$ of the parents would comment that the school must identify the week student's in all subjects and extended their support during the online classes could be beneficial for the weaker students to cope up with their studies. The majority $(65.4 \%)$ of the parents were expressing their thoughts that the individual subject teachers and schools must give a regular feedback about their child's performance during the online classes and this would be helpful for the parents to nurture their children accordingly.

Hence, the overall findings of the data analysis exposed the parents' perception on online learning system was timebeing, effective learning system during the crisis caused by COVID-19. However, it has also been revealed that parents were expected a regular school for the better monitor of their child's educational performance because the online learning tools may not be available to every learner everywhere.

\section{CONCLUSION:}

Covid -19 has made online classes unavoidable and the online learning is a distance learning system which requiring no physical presence of the learner in the traditional classroom. It is clear that this pandemic has completely wrecked the system of education. The present study was highlighting the parent's perception on virtual learning during covid pandemic. Though parents were facing lot of issues in their lives, the virtual learning platform is an additional burden for them in various aspects (monitoring, teaching, solve the technical issues etc). Even though government introduced a standardized protocol for the virtual classes, many schools have failed to follow which results children developed more psychological issues. To overcome such problems, multi-prolonged strategies are urgently needed in all school children to monitor their mental health levels. Since the technology and children education are coined together a well standardized syllabus should be introduced by the school education department to ensure the good health of the futuristic citizens of India.

\section{REFERENCES}

1. Cathy Li, and Farah Lalani, Entertainment and Sport Industries, World Economic Forum $20^{\text {th }}$ April 2020

https://www.weforum.org/agenda/2020/04/coronavirus-education-globalcovid19-online-digital-learning/ Accessed on 5.11.2020.

2. Rosenberg, Marc J. (2001).E-Learning Strategies for Delivering Knowledge in the Digital Age. McGraw-Hill, pp.3

3. Asma Khaleel Abdallah Dr., Parents Perception of e-learning in Abu Dhabi Schools, Abu Dhabi University, United Arab Emirates, International E-journal of Advances in Social sciences, vol.IV, Issue 10, April 2018,

http://ijasos.ocerintjournals.org Accessed 4.11.2020

4. Showkat Ahmad Dar Dr.Naseer Ahmad Lone, Journal of Indian Art History Congress ISSN: 0975-79 Volume-26, No.2(XIV): 2020-2021 47. https://www.researchgate.net...COVID-19

5. Eva Yi Hung Lau, Jian-Bin Li \& Kerry Lee, Online Learning and Parent Satisfaction during COVID-19: Child Competence in Independent Learning as a Moderator. Early Education and Development Volume 32, 2021 - Issue 6 Pages 830-842 | Published online:08 Jul 2021

6. Sonia Zaccoletti et.al., Parents' Perceptions of Student Academic Motivation during the COVID-19 Lockdown: A Cross-Country Comparison, Front.Psychol., 18 December 2020 | https://doi.org/10.3389/fpsyg.2020.592670

7. Ifoni Ludji, Tiarma Marpaung, Parents' Perception on the Implementation of Home Learning during Covid-19,

https://jbasic.org/index.php/basicedu/article/view/1013 DOI: https://doi.org/10.31004/basicedu.v5i5.1013

8. Andre Hasudungan Lubis et al., (2020) Parents Perception on E-Learning during Covid-19 Pandemic in Indonesia,

https://www.researchgate.net/publication/346615270parent\%27spercepti onsone-learningduringcovid-19pandemicinindonesia.

9. 16 Judy Amall, Problems with online/virtual learning; Excerpted from chapter 19 of Unschooling to University: Relationships matter most in a world crammed with content, posted on May3,2020

https://unschoolingtouniversity.com/2020/05/03/16-problems-withonline-virtual-school/ 
10. Role of Parents in online learning, 27 July 2020 by Vega Schools Education https://vega.edu.in/role-of-parents-in-online-learning/

11. Harkness, S, Super C. M., (1991). Parental Beliefs and Theories on Early Childhood Education. Journal Dev Psy,2: 193-202.

12. Graff, M., Davies, J., \& McNorton, M. (2008). Cognitive Style and CrossCultural Differences in Internet Use and Computer Attitudes. European Journal of Open, Distance and E-Learning.

13. UNESCO (2021) Adverse Consequences of school closures. https:// en.unesco.org/covid19/educationresponse/consequences 